\title{
Philosophiques
}

\section{Michel Paty, Einstein philosophe, Paris, Presses Universitaires de France (coll. " Philosophie d'aujourd'hui »), 1993, 584 pages.}

\section{Yvon Gauthier}

Volume 23, numéro 1, printemps 1996

Critères esthétiques et métamorphoses du beau

URI : https://id.erudit.org/iderudit/027383ar

DOI : https://doi.org/10.7202/027383ar

Aller au sommaire du numéro

Éditeur(s)

Société de philosophie du Québec

ISSN

0316-2923 (imprimé)

1492-1391 (numérique)

Découvrir la revue

Citer ce compte rendu

Gauthier, Y. (1996). Compte rendu de [Michel Paty, Einstein philosophe, Paris, Presses Universitaires de France (coll. « Philosophie d'aujourd'hui »), 1993, 584 pages.] Philosophiques, 23(1), 185-187. https://doi.org/10.7202/027383ar d'utilisation que vous pouvez consulter en ligne.

https://apropos.erudit.org/fr/usagers/politique-dutilisation/ 
Michel Paty, Einstein philosophe, Paris, Presses Universitaires de France (coll. "Philosophie d'aujourd'hui »), 1993, 584 pages.

C'est une véritable somme sur Einstein philosophe et sur la physique einsteinienne comme pratique philosophique que propose Michel Paty dans son récent livre. Michel Paty a en effet voulu, dans un patient effort, revoir l'œuvre entier d'Einstein dans la perspective de * la physique comme pratique philosophique * selon le sous-titre de l'ouvrage. 
Inspiré par le collectif Albert Einstein:philosopher-scientist, édité par Schilpp en 1949, l'A. a retracé pour son propre compte l'itinéraire philosophique d'Einstein, le parcours épistémologique (chap. VIII) d'un physicien qui avait formé une image personnelle du monde ("Weltbild »), d'après les mots d'Einstein lui-mème, davantage qu'une conception philosophique originale. On trouvera dans l'ouvrage une analyse historique détaillée, mais peu d'informations nouvelles sur le profil épistémologique d'Einstein qui se voyait comme épistérnologue ("Erkenntnistheoretiker ", p. 375). La bibliographie qui s'étend sur une soixantaine de pages suffirait à elle seule à assurer une place de premier choix parmi les ouvrages de référence sur Einstein ; on comparera à ce sujet la maigre biobibliographie scientifique d'Einstein de J. Merleau-Ponty (Einstein, Paris, Flammarion, 1993).

L'A. a choisi de ne pas faire l'exposé de la physique einsteinienne, de la Relativité Restreinte ou de la Relativité Générale. S'il n'est pas difficile de présenter la Relativité Restreinte sans trop de dètails techniques, la Relativité Générale exige une mise en forme plus sophistiquée et l'A. s'en est tenu ici au strict minimum; on le mesurera aux quelques mots qu'il a consacrés à la notion de connexion (p. 228), notion centrale s'il en est une dans la géométrie différentielle qui sert de support à la théorie générale de la relativité. L'A. a pris plutôt le parti du philosophe et après une introduction qui lui sert de justificatif, il entreprend de suivre l'ëlaboration de la pensée einsteinienne dans un ordre chronologique. La Relativité Restreinte fait lobjet des chapitres II, III et IV. L'analyse historique est fine et on retiendra les pages consacrées à la délicate question de l'importance qu'il faut accorder à l'expérience de Michelson-Morley dans la genèse de la Relativité Restreinte. Rappelons que l'expérience de Michelson-Morley visait à mesurer, à l'aide d'un interféromètre, l'effet d'entrainement d'un vent d'éther sur le mouvement de la Terre. Le résultat nul de l'expérience implique l'inexistence de l'éther. Einstein a semblé se contredire sur la question et l'A. opte pour une version moderée qui minimise l'importance de l'expérience, tout en indiquant que Einstein n'a peut-ètre pas reconnu à sa juste valeur le róle que l'expérience a pu jouer dans sa mise en cuvre de la théorie de la relativité. L'A. ne tient aucun compte de l'hypothèse récente selon laquelle la première épouse d'Einstein, Mileva Maric, serait sa source d'information sur lexpérience de Michelson-Morley. Mileva a en effet suivi les cours de Michelson à Heidelberg dans les années 1890 et a sans doute èté pour quelque chose dans les années de formation du jeune Einstein. Einstein s'est tu là-dessus et l'A. l'a imité.

Les chapitres V, VI, et VII portent sur l'extension à la Relativité Générale et à la question des rapports de la géométrie et de la physique, question qui a préoccupé Einstein au plus haut point. On sait que c'est la géométrie riemannienne qui a foumi à Einstein l'instrument mathématique pour la généralisation de la théorie de la relativité. Ici l'A. relit attentivement les textes d'Einstein et refait le trajet avec lui de Riemann et Helmholtz à Poincaré et Cartan. LA. s'en tient encore à Einstein et ne fait que quelques allusions aux travaux d'un Hermann Weyl dont l'ouvrage Raum, Zeit, Materie (1918) aura une influence considérable. C'est le principe de Mach, baptisé ainsi par Einstein luimème, qui sera le motif déterminant dans la constitution de la Relativité Générale. Encore là, Einstein a modifié sensiblement son appréciation du principe qui a connu une difficile intégration à la cosmologie. La critique de l'expérience du seau de Newton avait amené Mach à postuler que linertie d'un corps, sa masse inertielle est liée à la distribution de tous les corps dans l'univers. Il ne fait pas de doute que Einstein en a tiré l'essentiel de sa conception d'un univers sphérique fermé qui a été son modèle cosmologique 
privilégié (avec constante cosmologique pour garantir un univers statique). Einstein avouera plus tard que l'introduction du terme cosmologique dans les équations du champ aura été la plus grande erreur de sa vie.

Le chapitre VIII porte sur le parcours épistémologique d'Einstein et les influences philosophiques qu'il a subies. Kant, bien entendu, qu'il a lu tôt, mais aussi Hume, Poincaré, Mach. Mais Einstein n'est pas un philosophe pratiquant. c'est un physicien qui philosophe et il édifiera, comme nous l'avons dit plus haut, son image du monde à partir de fragments épars qu'il voudra unifier dans un conception générale de la science et de son objet.

Le dernier chapitre de l'ouvrage sur "Construction théorique et réalité " donne une idée assez juste du réalisme foncier de l'épistémologie einsteinienne. Einstein, en dépit des influences de Hume et de Kant, a toujours défendu une attitude réaliste qu'on ne peut qualifier de critique. Einstein a eu bien súr des velléités " constructivistes ", mais elles ne vont pas au-delà d'un kantisme bien tempéré. Le credo déterministe métaphorisé dans l'aphorisme "Dieu ne joue pas aux dés "s'accommodera mal de l'interprétation probabiliste de la Mécanique Quantique et les indications de l'A. sur la complêtude de la théorie physique à la fin de son ouvrage ne réussissent pas à justifier la dénégation épistémologique de la Mécanique Quantique chez Einstein. L'A. annonce un ouvrage sur Einstein, les quanta et le réel et il faudra attendre sa parution pour en évaluer la pertinence, mais on peut dire d'ores et déjà que la défense d'Einstein ira dans le sens d'un réalisme mitigé si l'on se fie aux remarques finales de l'A.

Le paradoxe d'Einstein-Podolsky-Rosen sur l'incomplétude de la Mécanique Guantique est le nœud gordien du réalisme einsteinien et on sait maintenant que les variables cachées locales (théorème de Kochen-Specher) ne suffisent pas à le trancher. La position de l'A. sur cette question semble se rapprocher de celle d'Einstein et il épouse à la fin une forme de réalisme modéré qui est plus un programme qu'une thèse philosophique bien définie.

On retiendra de cet ouvrage la lecture suivie de l'œuvre scientifique et parascientifique d'Einstein, l'analyse informelle des théories scientifiques et épistémologiques d'un physicien qui est devenu la figure populaire du savant au $\mathrm{XX}^{\mathrm{e}}$ siècle. Le souci d'exhaustivité fait de cette somme un outil de référence obligatoire dans le domaine français. Je ne relèverai qu'une coquille dans un travail qui en est généralement exempt, la formule de la note 3 . page 225, doit se lire :

$$
d s^{2}=d x_{1}^{2}+d x_{2}^{2}+d x_{3}^{2}-d x_{4}^{2}
$$

Les transcriptions de l'allemand comportent parfois des erreurs, en particulier, pages 392, 401, 402, 403,483. Mais ce ne sont là que des défauts mineurs dans un ouvrage dont il faut recommander la lecture aux philosophes des sciences et aux autres.

Yvon Gauthier

Département de philosophie Université de Montréal 